\title{
Présentation des remarques de Reggio (1612) sur le genre orné
}

\section{Anne Régent-Susini}

\section{(2) OpenEdition}

1 Journals

Édition électronique

URL : http://journals.openedition.org/rhetorique/709

DOI : 10.4000/rhetorique.709

ISSN : 2270-6909

Éditeur

UGA Éditions/Université Grenoble Alpes

Édition imprimée

ISBN : 978-2-37747-062-4

Référence électronique

Anne Régent-Susini, «Présentation des remarques de Reggio (1612) sur le genre orné », Exercices de rhétorique [En ligne], 11 | 2018, mis en ligne le 18 octobre 2018, consulté le 12 septembre 2020. URL: http://journals.openedition.org/rhetorique/709; DOI : https://doi.org/10.4000/rhetorique.709

Ce document a été généré automatiquement le 12 septembre 2020.

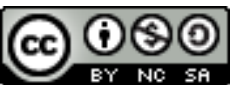

Les contenus de la revue Exercices de rhétorique sont mis à disposition selon les termes de la Licence Creative Commons Attribution - Pas d'Utilisation Commerciale - Partage dans les Mêmes Conditions 4.0 International. 


\title{
Présentation des remarques de Reggio (1612) sur le genre orné
}

\author{
Anne Régent-Susini
}

1 L'extrait présenté ici correspond au chapitre que le jésuite Carlo Reggio consacre au genre «exornatif», ou orné ${ }^{1}$, dans son monumental Orator christianus ( $1^{\mathrm{e}}$ éd. $1612^{2}$ ), somme rhétorique du début du XVII ${ }^{e}$ siècle consacrée à l'éloquence sacrée ${ }^{3}$, que Stefania Tutino considère comme "probablement le plus influent des traités de prédication catholique $\mathrm{du} \mathrm{XVII}{ }^{e}$ siècle $^{4} »$. Bien qu'enseignant au Collège Romain, centre du réseau éducatif jésuite, Reggio s'y adresse non à l'élève de collège, mais directement au prédicateur chrétien. Son traité accorde à la laudatio antique une place inédite dans la théorie de l'éloquence sacrée, promotion que Reggio s'emploie à justifier en invoquant de nombreuses autorités bibliques et patristiques et en développant une conception de l'éloge spiritualisée, marquée par l'influence de la mediocritas cicéronienne comme de la modestia chrétienne - loin des éloges flamboyants et efflorescents produits à la même époque par les jésuites français de l'entourage d'Henri IV et de Louis XIII, dont un Caussin, dans la grande synthèse des Eloquentiae sacrae et humanae parallela, fournit la théorie.

2 Reggio représente quant à lui, selon Marc Fumaroli, un représentant typiquement romain du " cicéronianisme dévot », qui, en s'inspirant du modèle cicéronien, tempère la sévérité des rhétoriques borroméennes par le recours à l'ornement. Dans son Orator christianus, il assimile donc, tout en les modérant par une influence cicéronienne réinterprétée à l'aune du christianisme, certaines leçons de Borromée sur la christianisation de la rhétorique : l'éloquence sacrée est pour lui avant tout un don de l'Esprit Saint ${ }^{5}$, mais l'orateur chrétien peut se préparer à le recevoir tant par la moralité de sa vie que par son étude de la théologie et des langues. Plus que sur l'inspiration, que valorisaient presque exclusivement les rhétoriques borroméennes, l'accent est placé sur l'imitation, directe (par l'écoute de bons prédicateurs) ou livresque (par la fréquentation de bons auteurs, à commencer par saint Paul ${ }^{6}$, suivi de Chrysostome, saint Grégoire et saint Bernard). L'héritage humaniste est clairement subordonné aux auteurs chrétiens, voire aux auteurs sacrés, dans la continuité desquels l'orateur 
chrétien, aide et auxiliaire de Dieu, doit inscrire sa parole. L'influence augustinienne, qui caractérisait auparavant les rhétoriques sacrées, se trouve en revanche minorée. C'est pourtant sur Augustin que s'appuie Reggio à l'orée du livre IV, afin de défendre la légitimité, pour l'orateur chrétien, d'un certain usage de la rhétorique. Non pas n'importe laquelle, cependant - car «il y a une vraie et une fausse éloquence " (" Quanam sit vera et falsa eloquentia $\left.{ }^{7} »\right)$; la seconde est notamment celle des sophistes dénoncés par Platon - et ce sont les Pères de l'Eglise qui permettront de distinguer l'une de l'autre ${ }^{8}$. Il s'agit donc de proposer une rhétorique du juste milieu, ne dédaignant pas l'ornatus (au contraire des rhétoriques borroméennes) tout en ne se livrant pas sans réserve aux modèles païens et à leurs grâces ornementales. L'enjeu est particulièrement délicat s'agissant du genre exornatif, qui vise à louer les actions vertueuses et s'illustre notamment par le panégyrique des saints : comme son nom l'indique en effet, c'est un genre intrinsèquement lié à l'ornement ${ }^{9}$, et par là particulièrement suspect de connivence avec les charmes trompeurs de la sophistique. Chez Reggio, le modèle cicéronien fonctionne moins comme une auctoritas que comme un principe régulateur.

3 L'un des traits originaux de l'ouvrage est le classement qu'il propose des sermons en trois types principaux, associés à trois types de public, classés selon une perfection croissante - en une typologie graduée qui s'inscrit dans une longue tradition chrétienne tentant de modéliser les étapes de l'union à Dieu. La tripartition sur laquelle s'appuie Reggio reflète en effet l'accord qui s'impose progressivement, à partir du Pseudo-Denys ( $v^{e}$ siècle ${ }^{10}$ ), sur les trois principales étapes de ce parcours de spiritualisation progressive : la voie purgative, la voie illuminative et la voie unitive ${ }^{11}$ correspondant dans une certaine mesure à la représentation ternaire de la personne en trois instances : le corps, l'âme et l'esprit ${ }^{12}$. Augustin retranscrit cette tripartition du point de vue des individus concernés, parmi lesquels il distingue les commençants, les progressants et les parfaits. Pour Reggio, le sermon « purgatif » combat certes les vices les plus répandus chez l'être humain, mais inclut également les discours visant à convertir les incroyants - qui constituent le commencement de ce parcours tripartite. Le sermon « illuminatif » développe la foi et exhorte à des vertus plus élevées. Enfin, le sermon « unitif » conduit les vertueux vers une plus grande perfection en traitant de sujets mystiques ou théologiques.

4 Reggio ne reprend donc pas la triade aristotélicienne des discours (judiciaire, délibératif et épidictique), mais il décompose au contraire sa propre trilogie (purgatif, illuminatif, unitif) en divers types de discours chrétiens :

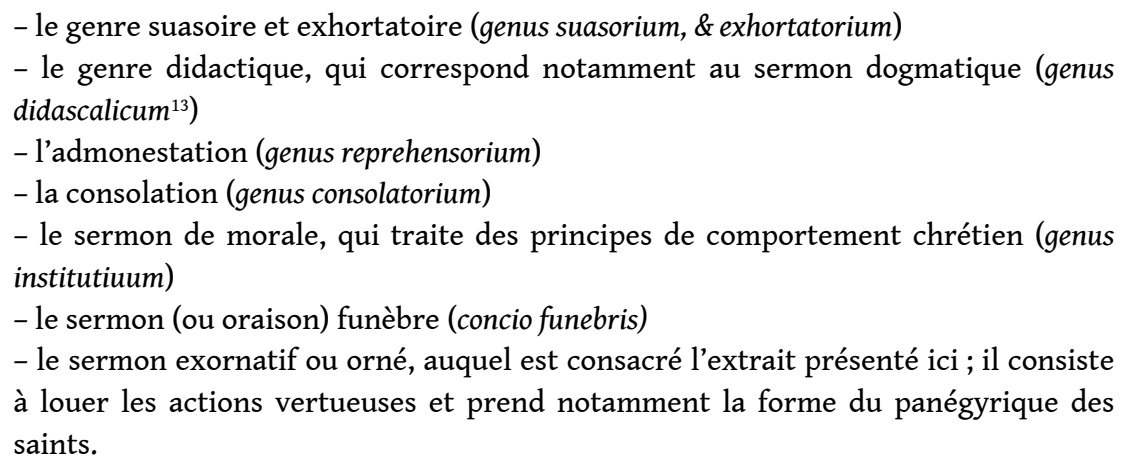

5 Ces catégories ne sont pas strictement étanches. On notera par exemple que, même si l'oraison funèbre n'est pas intégrée dans le sermon exornatif, mais plutôt définie comme un «mélange entre les genres exornatif, suasoire et quelquefois même 
didactique $^{14}$ ", certains des exemples avancés ici par Reggio, tels l'éloge composé par Grégoire de Nazianze pour saint Basile, renvoient bien à des discours funèbres, qui seront également convoqués au moment du développement tentant de fonder une concio funebris authentiquement chrétienne.

Quoi qu'il en soit, on le voit: Reggio passe sous silence l'héritage païen du genre épidictique, pour centrer son développement sur sa christianisation, reprenant la topique hagiographique des vertus et de leur hiérarchie, et invoquant les Pères et l'Ecriture elle-même. Cependant, on ne peut s'empêcher de juger quelque peu incantatoire cette énumération d'auctoritates exclusivement sacrées si l'on considère les discours effectifs relevant à l'époque de Reggio du genre exornatif, pétris d'humanisme et d'héritage antique ; la filiation tracée entre ces discours scripturaires et patristiques et l'éloge moderne demeure du reste fort peu spécifiée : c'est un horizon revendiqué, plus qu'un modèle formel à proprement parler. En fait, le développement proposé ici doit beaucoup - quoique cette dette demeure implicite - à la théorisation aristotélicienne du genre épidictique - tout en la reconfigurant dans une perspective chrétienne. On ne s'étonnera certes pas que, comme le genre épidictique dont il procède, le genre exornatif se caractérise par l'ornement, et en particulier l'amplification. Mais on notera en outre que l'homonoia propre au genre épidictique, qui, dans la plupart des cas, s'appuie sur la doxa et la renforce, tout en plaçant l'auditeur en position de spectateur, soit ici réinterprétée dans la perspective du cheminement vers Dieu. C'est que, comme les sermons dogmatiques, moraux, de conseil, de consolation, le sermon exornatif (ou orné) appartient, on l'a vu, aux discours " unitifs ». Or, ceux-ci s'adressent à un public qui adhère déjà aux vérités chrétiennes et pour qui la progression morale et spirituelle passe par le spectacle des vertus, qui leur permettra ultimement de se reposer en Dieu, voire de s'unir à Lui dans une commune contemplation du Bien - la position de spectateur étant, comme on sait, celle qui distingue radicalement, chez Aristote, l'auditeur du discours épidictique des auditeurs des discours judiciaire et délibératif.

7 C'est donc, comme le précise d'emblée Reggio, du spectacle de la vertu que procédera la valeur exhortative du discours. Mais ce spectacle procure d'abord à l'orateur lui-même une jouissance qui semble conjointement morale, spirituelle et esthétique : contempler la vertu incarnée dans des vies de saints produit, outre l'éloge lui-même, l'admiration pour la bonté de Dieu qui s'exprime par celle des créatures, et l'encouragement à l'imitation, puisque les saints, étant hommes donc marqués par le péché, n'en ont pas moins réussi à manifester de hautes vertus : le sombre thème augustinien de la massa peccati (littéralement, la pâte ou l'argile du péché) est renversé en une exhortation confiante. L'éloge, tel qu'il se trouve ici théorisé par Reggio, a donc deux objets : le saint (ou la vertu) considéré(e), et Dieu lui-même. C'est à l'éloge de ce dernier, en tant que source de toute vertu, qu'est subordonné tout le discours, et en particulier l'éloge du saint. C'est donc un éloge, en quelque sorte, "à double détente ", que produit l'orateur chrétien, éloge dont le fonctionnement à la fois atteste et reproduit une éthique et une spiritualité de la médiation.

8 Deux autres éléments au moins signalent la rupture qu'introduit la perspective chrétienne dans la théorisation païenne de l'épidictique. D'une part, Reggio insiste pour finir sur la possibilité de louer non seulement des personnes et des actions, mais des vertus - en une démarche peu fréquente, même dans la Seconde Sophistique, où les 
éloges de personnes, toujours prédominants, voisinent avec les éloges de cités, de dieux ou d'objets, mais guère avec des éloges de vertus en tant que telles.

D'autre part et surtout, Reggio met en place une topique de l'éloge. Le chapitre 10 que nous éditons s'intitule en effet exactement "Les lieux dans le genre orné », après deux ensembles consacrés, l'un aux lieux « topiques » et l'autre aux lieux « théologiques ${ }^{15}$ ». Ce renouvellement de la topique est sensible en particulier à travers le traitement du lieu du genus (la noblesse de l'extraction), fondamental dans les éloges de personnes. Par là, il prend explicitement ses distances avec les topoi païens de l'éloge : "Les rhéteurs ont coutume de présenter plusieurs lieux de la louange [...]. Notre orateur toutefois n'est pas astreint à ces lieux, et pour certains, c'est à peine s'il doit les aborder. » Il ne s'agit certes pas de répudier tous ces lieux - mais ceux qui seront conservés feront l'objet d'un réinvestissement proprement chrétien, à commencer par le lieu du genus (noblesse de l'extraction, de la «lignée»), traditionnellement essentiel et même littéralement primordial, dans tout éloge de personne. Les Pères fournissent à Reggio deux directions pour ce réaménagement : Grégoire de Nazianze, le plus radical, considère que la noblesse, qui désigne pour lui «le fait de conserver l'image et la ressemblance de Dieu ", "doit être pesée et estimée à titre individuel et à l'aune des mérites de chacun». Ambroise, en revanche, transpose le lieu du genus dans une perspective chrétienne : «Saint Ambroise, lui, dans son commentaire sur Luc, enseigne en s'appuyant sur les livres sacrés que chez ceux qui méritent l'éloge il convient de louer non seulement les mœurs, mais encore leurs parents. [...] Toute noblesse profane doit donc être méprisée plutôt qu'encensée par l'orateur chrétien ; en revanche, celle qui procède de la vertu des parents peut à bon droit faire l'objet d'une louange. » Pour Ambroise, ce n'est pas la fausse noblesse, celle du statut social, qui se transmet héréditairement, mais la véritable, c'est-à-dire la noblesse morale. On notera cependant que cette méfiance envers les qualités héritées n'était pas totalement absente chez les théoriciens antiques, à commencer par Aristote, qui considère que seuls les actes volontaires sont dignes de louange ${ }^{16}$, et Quintilien, pour qui seul l'usage « honnête » des avantages reçus justifie la louange, l'éloge de l'âme (ou du caractère), siège de la volonté, étant toujours véritable ( animi semper vera laus ${ }^{17} »$ ). Mais les auctoritates absentes sont ici aussi significatives que les présentes. Contre une représentation implicite de l'éloge comme quintessence de la rhétorique païenne, de ses valeurs et de ses ornements mensongers, il s'agit bien en effet d'autoriser un éloge authentiquement chrétien - dont le modèle ultime peut être trouvé dans les Écritures elles-mêmes, qui comportent des louanges - louanges des justes, mais également louanges de Dieu. Comment, dès lors, l'éloge n'appartiendrait-il pas de plein droit à la rhétorique chrétienne?

10 Aussi les auctoritates exclusivement scripturaires et patristiques (Grégoire de Nazianze, Basile de Césarée, saint Cyprien, saint Jérôme), qu'invoque Reggio, soulignent-elles tout autant qu'elles justifient l'inflexion délibérément chrétienne de son exposé: ni les grands théoriciens ni les grands praticiens des éloges païens ou humanistes ne sont mentionnés dans cet exposé visant à fonder sur des bases résolument chrétiennes une théorie de l'éloge susceptible d'exalter la « vraie » vertu - c'est-à-dire, en définitive, le vrai Dieu. L'extrait ici traduit offre ainsi à la fois un jalon et un exemple remarquable dans la christianisation rhétorique de l'éloge qui s'opère dans l'Europe de la première modernité. À travers le traitement réservé au lieu du genus notamment, il illustre la manière dont l'éloge, fût-il inséparable d'un certain conservatisme (rançon inévitable 
de son rapport privilégié au consensus), peut, dans certaines circonstances, œuvrer à une modification des cadres axiologiques.

\section{NOTES}

1. Sur le lien entre exornatio, ornement et épidictique, voir dans ce même numéro d'Exercices de rhétorique, l'article de Cécile Lignereux, «L'éloge, maillon faible de la lettre de remerciement? ", n. 43. Dans certains contextes, l'exornatio s'identifie même avec l'amplificatio.

2. Carlo Reggio, Orator christianus, Rome, Bartholomeus Zanettus, $1612,2^{\mathrm{e}}$ partie, livre 4, chap. 10 : «In genere exornativo loci », p. 284-286.

3. Marc Fumaroli considère que son pendant profane est l'ouvrage de Famiano Strada intitulé Prolusiones Academicae (1617). Voir M. Fumaroli, L'Âge de l'éloquence, Paris, Albin Michel, 1994, p. 186.

4. Stefania Tutino, Shadows of Doubt, New York, Oxford University Press, 2014, p. 138.

5. Sur la métaphore musicale filée par Reggio, qui compare le prédicateur à un instrument de musique entre les mains de Dieu, voir S. Conte, «Saint Paul et saint Jean Chrysostome modèles des rhétoriques sacrées de Carlo Reggio et de Nicolas Caussin ", dans G. Vagenheim et M. Deramaix éd., L'Italie et la France dans l'Europe latine du XIV ${ }^{e}$ au XVII siècle - Influence, émulation, traduction, Rouen, Presses Universitaires de Rouen, 2007, p. 307-321, ici p. 308.

6. Voir Carlo Reggio, Orator christianus, op. cit., III, 20, p. 181-190 : «Exemplar Concionatorum Paulus, et erga eum devotio "; et S. Conte, op. cit.

7. Carlo Reggio, Orator christianus, op. cit., IV, 4, p. 203.

8. Voir Carlo Reggio, Orator christianus, op. cit., IX, 38, p. 700.

9. Sur le lien privilégié que conserve l'ornement avec l'éloge (contrairement au blâme) à la période moderne, voir par exemple la magistrale étude de François Cornilliat portant sur les "Grands rhétoriqueurs", prédécesseurs et contemporains de Marot (Or ne mens: couleurs de l'éloge et du blâme chez les "grands rhétoriqueurs", Paris, H. Champion, 1994).

10. L'influence dionysienne, très forte au Moyen Âge, survit sans trop de mal aux progrès philologiques et critiques de la Renaissance et du premier XVII ${ }^{\mathrm{e}}$ siècle, en dépit des problèmes de plus en plus grands que pose la figure du pseudo-Denys, superposition mythique de trois figures " historiques » : le magistrat de l'Aréopage converti par saint Paul dans les Actes des Apôtres, l'auteur du corpus dionysien et le premier évêque de Paris, martyrisé au III ${ }^{\mathrm{e}}$ siècle, considéré comme l'« apôtre de la France ». Voir Y. De Andia, Denys l'Aréopagite et sa postérité en Orient et en Occident, Paris, Institut d'Études Augustiniennes, 1997 ; et J.-M. Le Gall, Le Mythe de saint Denis entre Renaissance et Révolution, Seyssel, Champ Vallon, 2007. Le Pseudo-Denys est cité à plusieurs reprises dans l'Orator christianus ; voir par exemple éd. citée, p. 256-257.

11. Sur la reprise de cette structure dionysienne chez Thomas d'Aquin, voir Somme théologique, IIII, 163:4. Et sur sa réinterprétation par le grand scolastique jésuite espagnol Francisco Suárez, voir, de cet auteur, De Religione, Lyon, Cardon et Cavellat, 1620, tr. VIII, livre I, chap. 13.

12. Sur l'évolution des conceptions médiévales, d'abord principalement ternaires, vers des formulations plus binaires, voir cependant J. Baschet, « Âme et corps dans l'Occident médiéval : une dualité dynamique, entre pluralité et dualisme », Archives de sciences sociales des religions, 112 | 2000, p. 5-30 [article repris dans Corps et âmes: une histoire de la personne au Moyen Âge, Paris, Flammarion, 2016]. 
13. Ou didascalium : Reggio recourt aux deux graphies; il nomme encore genus doctrinale ce genre créé par Melanchthon.

14. Ph. Casarotto, «Les oraisons funèbres impériales en Autriche de Ferdinand II à Charles VI (1637-1740): discours et représentations ", thèse de doctorat dirigée par J.-M. Valentin, Université Paris IV-Sorbonne, 2004, thèse dactyl., $1^{\mathrm{e}}$ partie. Je remercie vivement Philippine Casarotto de m'avoir généreusement facilité la consultation de ses travaux.

15. «Loci Topici », chapitres 4 et 5, p. 266-273 : les lieux de la définition, de l'étymologie, etc. « Loci Theologici ", chapitres 6 à 9, p. 273-284 : les lieux des témoignages (et de l'autorité divine), de la tradition, etc. Le chapitre 10 est lui-même le début d'un nouvel ensemble : les lieux du genre orné (chap.10), puis on va des lieux du genre suasoire à ceux de l'oraison funèbre, selon l'ordre indiqué plus haut (chap. 11 à 17, p. 287-309).

16. Voir Aristote, Rhétorique, I, 9, 1366a, 33-36.

17. Quintilien, Institution oratoire, III, 7, 14-15: «L'éloge du caractère est toujours vrai » (trad. Cousin, Paris, Les Belles Lettres, C. U. F., 1976).

\section{AUTEUR}

\section{ANNE RÉGENT-SUSINI}

Université Sorbonne nouvelle - Paris 3 